\title{
In Vitro Model Systems to Investigate Drug Resistance Mechanisms in Pancreatic Cancer Cells
}

\author{
Eric Romney ${ }^{1}$, Vinay J. Nagaraj ${ }^{*}$ \\ ${ }^{1}$ Arizona College of Osteopathic Medicine, Midwestern University, Glendale, Arizona \\ ${ }^{2}$ Department of Biochemistry, Midwestern University, Glendale, Arizona \\ Email: eromney92@midwestern.edu, *nvinay@midwestern.edu
}

Received 25 November 2015; accepted 27 December 2015; published 30 December 2015

Copyright (C 2015 by authors and Scientific Research Publishing Inc.

This work is licensed under the Creative Commons Attribution International License (CC BY). http://creativecommons.org/licenses/by/4.0/

(c) (i) Open Access

\section{Abstract}

With a 5-year survival rate of less than $6 \%$, the diagnosis of pancreatic cancer is devastating news for any patient. Gemcitabine, the most commonly used chemotherapy drug, only improves survival by approximately 1.5 months. A major obstacle to the treatment of pancreatic cancer with gemcitabine is the development of drug resistance. To better understand the precise mechanisms by which patient tumor cells gain resistance to gemcitabine, a cell culture model system that more accurately reflects the development of drug resistance in vivo is required. In this study, cultured pancreatic adenocarcinoma BXPC-3 cells were subjected to two different treatment regimens. The first method-termed pulse method-involves periodically treating separate cultures of BxPC-3 cells with constant predetermined doses of gemcitabine. The second treatment regimen-termed incremental method-consists of treating BxPC-3 cells with increasing doses of gemcitabine from 10 to $100 \mathrm{nM}$. While all treated cells showed enhanced resistance to gemcitabine, low-dose pulse treatments were sufficient to produce highly drug-resistant cells as evidenced by higher IC $_{50}$ measurements. Pulse treatments also resulted in slower growth rates and increased doubling time of the drug-resistant cells. Morphological changes indicate cellular abnormalities linked to likely epithelial-to-mesenchymal transition and drug resistance. Our preliminary results indicate that the pulse method may better simulate resistance observed in patients undergoing chemotherapy and may serve as a superior model to investigate drug-resistance. This model can also help with identification of appropriate markers that determine the presence of drug-resistant cells and help clinicians adjust treatment strategies to improve outcomes for patients suffering from pancreatic cancer.

\section{Keywords}

Pancreatic Cancer, Gemcitabine, Drug-Resistance, Pulse Method, Incremental Method

\footnotetext{
${ }^{*}$ Corresponding author.
}

How to cite this paper: Romney, E. and Nagaraj, V.J. (2015) In Vitro Model Systems to Investigate Drug Resistance Mechanisms in Pancreatic Cancer Cells. Advances in Biological Chemistry, 5, 286-292. 


\section{Introduction}

In the United States, pancreatic cancer is the $5^{\text {th }}$ most deadly cancer with an estimated 40,560 deaths out of the 48,960 people that were diagnosed with this disease in 2015 [1]. In spite of the great amount of research on pancreatic cancer over the last few decades, there has only been a marginal increase in the 5-year survival rate from $2 \%$ for $1975-1977$ to $6 \%$ for 2002-2008 [2]. The exceptionally poor prognosis for pancreatic cancer can be attributed to several factors. Among them, late diagnosis of the diseases seems to be an important factor [2] [3]. A study of patients from 2002-2008, shows that as many as $90 \%$ of the pancreatic cancers that could be staged had either regional or distant metastases at the time of diagnosis [2]. While early detection of pancreatic cancer is critical to improve patient outcomes [4], there is an urgent need to develop more effective chemotherapies [5]. In 1997 gemcitabine (GEM), a nucleoside analog, replaced fluorouracil (5-FU) as the standard of care for patients with metastatic pancreatic cancer and continues to be the chemotherapeutic of choice to date [5] [6]. Since that time, different chemotherapeutic combination strategies have been used clinically with marginal effects on survivability [5]. Most of these therapies have included gemcitabine as a chemotherapeutic backbone combined with another drug to enhance its effects [5] [7].

Development of acquired drug resistance is a significant factor for the poor prognosis of patients with pancreatic cancer during gemcitabine chemotherapy [8]. There are indications that the development of resistance to gemcitabine and other chemotherapeutics, in part, is also responsible for the development of pancreatic cancer metastasis by encouraging an epithelial-mesenchymal transition (EMT) in cancer cells [9]-[11]. This is important because it is the widespread metastases that are blamed for the rapid progression of the clinical disease leading to mortality. In addition, there have been several studies regarding pancreatic cancer stem cells and the possible role these cell subpopulations may play in the development of drug resistance [12]-[18]. Interestingly, as few as 100 pancreatic cancer stem cells appear to be enough to form a new tumor in a mouse model [17]. Based on this new understanding of pancreatic adenocarcinoma tumor biology, we sought to explore in vitro the mechanisms by which patient tumor cells gain resistance to gemcitabine during their chemotherapy. This knowledge is key to identifying novel biomarkers as well as developing therapies that can prevent resistance from ever occurring.

In vitro techniques to create drug resistant cancer cells in the lab vary considerably. Previous studies on gemcitabine resistance most commonly use an "incremental method" where cultured pancreatic cancer cells receive increasing doses of gemcitabine in a step-wise manner. Typically, time is allowed for cell recovery in between treatments, and gemcitabine doses provided to cells can reach up to 50 times the initial $\mathrm{IC}_{50}$ [19] [20]. A lesser used method that has not been previously used on pancreatic cancer is the "pulse method" [21]. In this method, cells are given a metronomic dose of the chemotherapeutic agent with time to recover in between treatments. Preliminary results from ovarian cancer cells indicate that this method is more similar to the standard dosing regimen used on patients undergoing treatment [21]. In this short report, we compared the incremental and pulse methods using cultured human pancreatic adenocarcinoma cell line BxPC-3 with the goal of better modelling the resistance process that occurs in vivo during gemcitabine treatment.

\section{Results and Discussion}

\subsection{Development of Drug-Resistance in Response to Different Drug Treatment Methods}

Currently, there are a wide variety of methods used by researchers to induce drug resistance in pancreatic cancer cells but a variation of the incremental method described by Shah et al. [18] [20] is the most frequently used. This method involves choosing an initial low dose and exposing the cells to that dose for a given length of time. After one or more treatments at that initial dose, the drug is increased by some factor and the cycle is repeated. This is in contrast to a lesser used method known as the "pulse method" [21]. The pulse method is designed to closely resemble the weekly intravenous pulse dosing regimen of gemcitabine administered to pancreatic cancer patients. Specifically, cells are periodically exposed to an unchanged dose without increasing its initial concentration. We compared the two different treatment protocols with the end goal of determining if there is a better clinical model to determine pharmacodynamic biomarkers of response and resistance to gemcitabine during pancreatic cancer therapy.

For this study, cultured pancreatic adenocarcinoma BxPC-3 cells were subjected to the two different treatment regimens. The first treatment regimen consists of treating BxPC-3 cells with increasing doses of gemcitabine from 10 to $100 \mathrm{nM}$ (Table 1). The second method involves periodically treating separate cultures of BxPC-3 
Table 1. Treatment schedule for the development of gemcitabine resistance in the pancreatic adenocarcinoma cell line BxPC-3. Gemcitabine treatments (indicated in $\mathrm{nM}$ ) were administered when the cells reached $50 \%$ confluence in the tissue culture flask. After incubation of the cells with the drug for two days, the cells were allowed to recover in drug-free media until they reached $70 \%$ confluence. At this stage, cells were passaged and the next treatment was commenced at 50\% confluency.

\begin{tabular}{|c|c|c|c|c|c|c|c|c|c|c|}
\hline \multicolumn{11}{|c|}{ Gemcitabine treatment schedule } \\
\hline Treatment number & 1 & 2 & 3 & 4 & 5 & 6 & 7 & 8 & 9 & 10 \\
\hline Control BxPC-3 (0 nM) & 0 & 0 & 0 & 0 & 0 & 0 & 0 & 0 & 0 & 0 \\
\hline Incremental (10 - 100 nM) & 10 & 20 & 30 & 40 & 50 & 60 & 70 & 80 & 90 & 100 \\
\hline Pulse (60 nM) & 60 & 60 & 60 & 60 & 60 & 60 & 60 & 60 & 60 & 60 \\
\hline Pulse (30 nM) & 30 & 30 & 30 & 30 & 30 & 30 & 30 & 30 & 30 & 30 \\
\hline Pulse (15 nM) & 15 & 15 & 15 & 15 & 15 & 15 & 15 & 15 & 15 & 15 \\
\hline
\end{tabular}

cells with constant predetermined doses of gemcitabine (Table 1). Using these different protocols we developed 4 different gemcitabine-resistant subcultures of BxPC-3 cells and compared their characteristics (Figure 1).

Our results indicate that as few as 10 treatments of gemcitabine are sufficient to create highly drug-resistant BxPC-3 subcultures (Figure 1). All of the subcultures showed a two-fold or higher increase in resistance to gemcitabine despite large differences in maximum treatment dose (15 $\mathrm{nM}$ to $100 \mathrm{nM})$ and treatment regimen. Gemcitabine $\mathrm{IC}_{50}$ measurement increased significantly from about $30 \mathrm{nM}$ for the parental BxPC-3 cells to about 55 - $80 \mathrm{nM}$ for the gemcitabine treatments. Figure 1(A) through Figure 1(E) show representative dose response curves of 3 independent experiments for gemcitabine which were used for calculating $\mathrm{IC}_{50}$ measurements.

It is very interesting to note that pulse treatment at the lowest dose $(15 \mathrm{nM})$ leads to the greatest drug resistance ( $\mathrm{IC}_{50}$ of $\sim 80 \mathrm{nM}$ ). We hypothesize that exposure to the pulse treatment, especially at sub-lethal doses, may allow cancer cells to better activate molecular pathways for drug resistance. These may include drug-efflux mechanisms, drug degradation pathways, intracellular drug sequestration mechanisms, alterations to the drug target, activation of repair mechanisms to undo drug damage, etc. A molecular analysis of each of these mechanisms is necessary to understand their contribution to the development of drug resistance in the BxPC-3 subcultures.

\subsection{Effects of Incremental and Pulse Treatments on the Growth of BxPC-3 Cells}

We compared the growth rates of BxPC-3 subcultures derived by pulse and incremental methods to identify if there are any differences. Previous studies with ovarian cancer cells have found that drug-resistant subcultures have a decreased rate of proliferation [21]. We report similar findings with BxPC-3 pancreatic adenocarcinoma cells. The pulse-treated cells, in particular, showed a greater decrease in cell proliferation (Figure 2(A)). The incremental method yielded only a $19.4 \%$ increase in doubling time whereas the $15 \mathrm{nM}$ pulse and $60 \mathrm{nM}$ pulse showed an increase of $45.4 \%$ and $60.2 \%$ respectively (Figure 2(B)). We believe these results may show clinically relevant differences between the incremental and pulse methods. Since chemotherapeutic agents typically target fast-growing cells in the body, a reduction in the doubling time is likely to help the drug-resistant cells evade some of the effects of gemcitabine and contribute to drug-resistance.

\subsection{Drug-Resistance-Induced Changes in the Morphology of Cells}

Throughout the gemcitabine treatment protocols, cells were observed and imaged to document any morphological changes. During that time, we specifically looked for any morphological changes related to metastasis and signs of possible epithelial-mesenchymal transitions (EMT) [9] [10] [20] [21]. Gemcitabine-sensitive (parental) BxPC-3 cells grow in colonies with a typical epithelial cobblestone appearance (Figure 3(A)). During gemcitabine treatment, two new morphologies were observed in both the incremental and pulse dosing regimen. The first morphology was very large isolated cells after gemcitabine exposure (Figure 3(B)). These cells were more prominent in the pulse treatments, appeared to increase in number at later treatments, and persisted for at least 10 cell culture passages after the last treatment. The significance, if any, of these cells is unknown as these cells have not been previously described in the context of drug-resistance. Studies with ovarian cancer cells hypothesize that these enlarged cells may contain vesicles for the intracellular sequestration of the chemotherapeutic 
agent [21]. Since the drug resistant cells show a slower growth rate-another possibility is that the accumulation of cellular components in preparation for cell division, with a delay in the progression of the cell cycle, may also lead to large sized cells.

The second phenotype is the spindle-shaped cells with long neuron-like morphology (Figure 3(C)). This morphology seemed to appear after 3 treatments and were observed in all the different drug treatments. The proportion of these cells were higher when the cell cultures were at lower confluency and decreased significantly

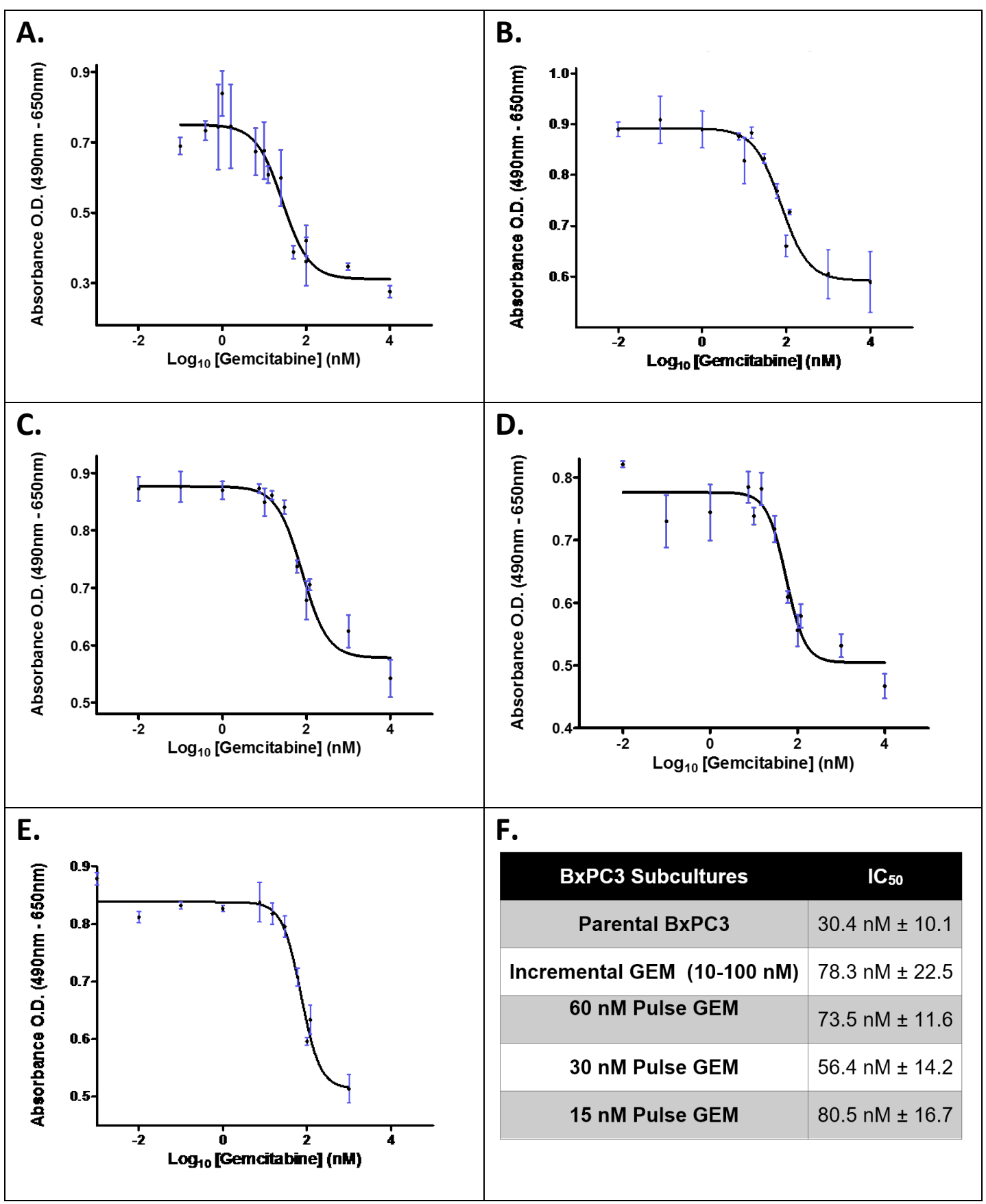

Figure 1. (A) Shows the dose response curves of the parental (untreated) BxPC-3 cells to gemcitabine and that of the BxPC-3 cells exposed to gemcitabine by 10 - $100 \mathrm{nM}$ incremental treatment (B), $15 \mathrm{nM}$ pulse treatment (C), $30 \mathrm{nM}$ pulse treatment (D) and $60 \mathrm{nM}$ pulse treatment (E). The $\mathrm{IC}_{50}$ for each BxPC-3 subculture following treatments with gemcitabine is the shown in the table in (F). 


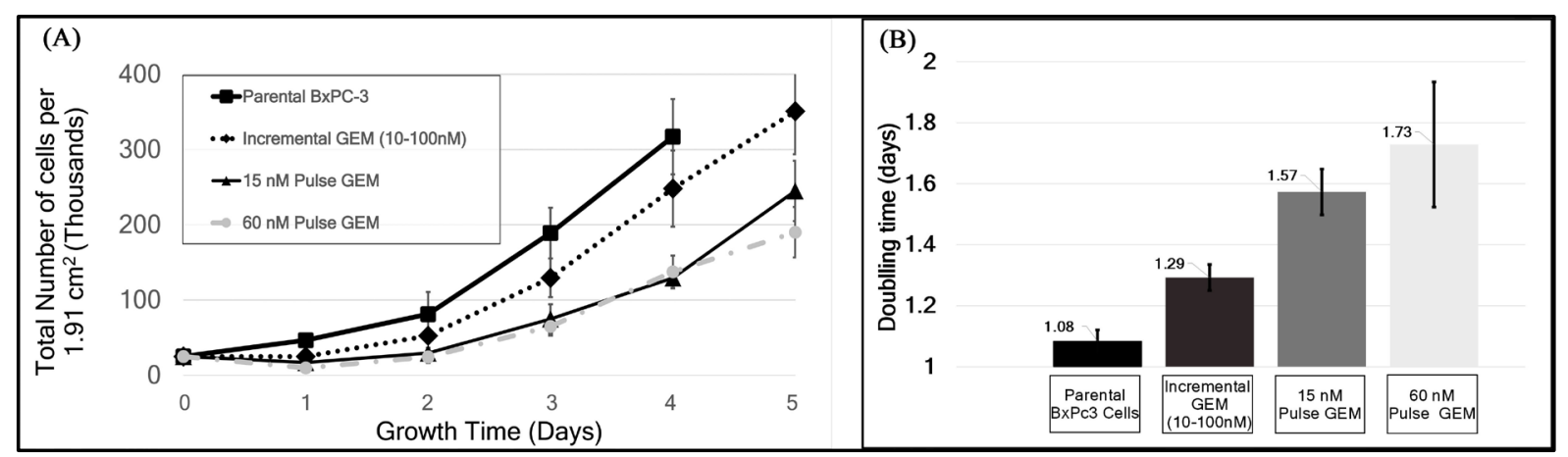

Figure 2. (A) Growth curves for the parental BxPC-3 cell line and gemcitabine-resistant BxPC-3 subcultures; (B) Doubling times for the parental BxPC-3 cell line and gemcitabine-resistant BxPC-3 subcultures.

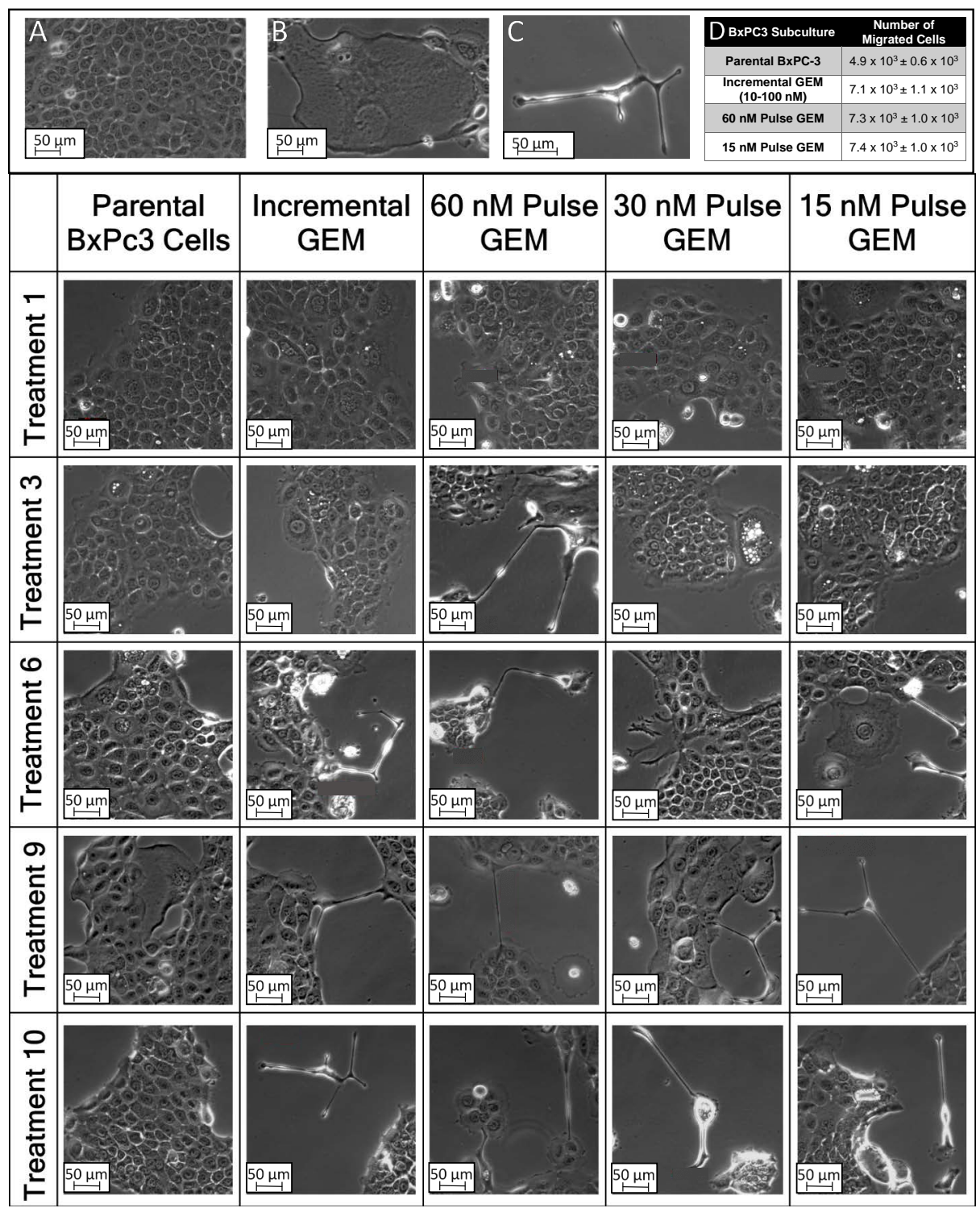

Figure 3. (A), (B), (C) and (E) Morphological changes observed in pancreatic cancer BxPC-3 cells during the development of resistance to gemcitabine; (D) Table shows the number of migratory cells in a Boyden chamber-based cell migration assay that was performed to assess the metastatic potential of BxPC-3 subcultures. 
at higher confluences. We believe that these cells may be responsible for the increase in migration and metastasis observed in vivo in patients undergoing chemotherapy. Moreover, their transient nature fits with the current "seed and soil" model of metastasis which is said to require an epithelial-mesenchymal transition (EMT) to leave the dense stroma of the pancreas. However a reversal of this phenotype i.e. mesenchymal-epithelial transition (MET) is required to complete metastasis [8]-[11] [22]. In order to determine the metastatic potential of the drug-treated cells, a Boyden chamber-based migration assay was performed. All drug-resistant subcultures showed a significant increase in the number of cells able to migrate through the $8 \mu \mathrm{m}$ pores when compared with the parental BxPC-3 cell line used as a control (Table 1, Figure 3). If we take into account the relatively slower growth rate of the resistant subcultures, actual migratory potential of the pulse-treated cells may be even higher than what has been experimentally determined. It appears that drug-resistance is strongly associated with enhanced metastatic potential of the cancer cells.

\section{Conclusions}

Pancreatic cancer is a deadly disease with a very high rate of mortality. Drug resistance is a major reason for the failure of chemotherapy and results in numerous deaths from pancreatic cancer. Previous research on the mechanisms of drug-resistance in cancer cells in the lab uses the incremental dose method to create drug-resistant cells. Although popular for studying resistance, these studies by design may not appropriately reflect the development of drug-resistance in vivo. In the incremental method, drug-resistant cells are selected by treatment with extremely high concentrations of the drug. A consequence to this approach may be the elimination of subpopulation of cells that may display other clinically relevant properties and phenotypes important for drug resistance, metastasis and occurrence of cancer stem cells.

Previous studies have indicated that chemoresistant cells are more metastatic [8] [19] [21]. Gemcitabine-resistant cells in the current study displayed strong migratory capabilities even with a low-dose pulse treatment. Future studies investigating changes in E- and N-Cadherins and other EMT/metastases markers will shed more light on this subpopulation of cells.

Our preliminary results revealed significant differences in the growth rates between the pulse and incrementally treated cells. This may suggest—in part—a greater presence of cancer stem cell population in the pulse treatment subcultures versus the traditional incremental method [12]. Cells triple positive for CD44, CD24 and epithelial cell adhesion molecule (ESA, CD326, EpCAM) will confirm the presence of pancreatic cancer stem cells [17]. Other markers which indicate pancreatic cancer stem cell-like properties that need to be examined include CD184 (CXCR4) and CD133, and the more recently described CD24, and CD338 (ABCG2) [18]. Drugresistant cells derived from this study can also be useful to identify other novel determinants of pancreatic cancer stem cells.

In conclusion, we have shown that, the chemo-resistance process can occur in vitro with periodical exposure to a minimal amount of drug. In addition to drug-resistance, gemcitabine treatment may induce metastases while also enriching the tumor with cancer stem cells. Indeed, our observations seem to match the actual clinical course for patients treated with gemcitabine. Patients experience an initial decrease in tumor burden only to succumb months later to metastasis. Further research using the pulse method on cell lines may provide more clinically relevant information on these cellular/molecular processes and identify appropriate markers that determine drug-resistance. This knowledge will help clinicians adjust treatment strategies to improve outcomes for patients suffering from pancreatic cancer.

\section{Acknowledgements}

We thank Bernardo Chavira, Whitney Wilson, Zachary Emmons and Omar Jawhar for technical help. Eric Romeny was supported by the Kenneth A Suarez research fellowship.

\section{References}

[1] Siegel, R.L., Miller, K.D. and Jemal, A. (2015) Cancer Statistics, 2015. CA: A Cancer Journal for Clinicians, 65, 5-29. http://dx.doi.org/10.3322/caac.21254

[2] Siegel, R., Naishadham, D. and Jemal, A. (2013) Cancer Statistics, 2013. CA: A Cancer Journal for Clinicians, 63, 1130. http://dx.doi.org/10.3322/caac. 21166 
[3] Jemal, A., Bray, F., Center, M.M., Ferlay, J., Ward, E. and Forman, D. (2011) Global Cancer Statistics. CA: A Cancer Journal for Clinicians, 61, 69-90. http://dx.doi.org/10.3322/caac.20107

[4] Paulson, A.S., Tran Cao, H.S., Tempero, M.A. and Lowy, A.M. (2013) Therapeutic Advances in Pancreatic Cancer. Gastroenterology, 144, 1316-1326. http://dx.doi.org/10.1053/j.gastro.2013.01.078

[5] Michl, P. and Gress, T.M. (2013) Current Concepts and Novel Targets in Advanced Pancreatic Cancer. Gut, 62, 317326. http://dx.doi.org/10.1136/gutjnl-2012-303588

[6] Burris 3rd, H.A., Moore, M.J., Andersen, J., et al. (1997) Improvements in Survival and Clinical Benefit with Gemcitabine as First-Line Therapy for Patients with Advanced Pancreas Cancer: A Randomized Trial. Journal of Clinical Oncology: Official Journal of the American Society of Clinical Oncology, 15, 2403-2413.

[7] Kothari, N., Saif, M.W. and Kim, R. (2013) First-Line Treatment for Advanced Pancreatic Cancer. JOP: Journal of the Pancreas, 14, 129-132.

[8] Long, J., Zhang, Y., Yu, X., et al. (2011) Overcoming Drug Resistance in Pancreatic Cancer. Expert Opinion on Therapeutic Targets, 15, 817-828. http://dx.doi.org/10.1517/14728222.2011.566216

[9] Arumugam, T., Ramachandran, V., Fournier, K.F., et al. (2009) Epithelial to Mesenchymal Transition Contributes to Drug Resistance in Pancreatic Cancer. Cancer Research, 69, 5820-5828. http://dx.doi.org/10.1158/0008-5472.CAN-08-2819

[10] Wu, Q., Miele, L., Sarkar, F.H. and Wang, Z. (2012) The Role of EMT in Pancreatic Cancer Progression. Pancreatic Disorders \& Therapy, 2. http://dx.doi.org/10.4172/2165-7092.1000e121

[11] Sarkar, F.H., Li, Y., Wang, Z. and Kong, D. (2009) Pancreatic Cancer Stem Cells and EMT in Drug Resistance and Metastasis. Minerva Chirurgica, 64, 489-500.

[12] Hermann, P.C., Huber, S.L., Herrler, T., et al. (2007) Distinct Populations of Cancer Stem Cells Determine Tumor Growth and Metastatic Activity in Human Pancreatic Cancer. Cell Stem Cell, 1, 313-323. http://dx.doi.org/10.1016/j.stem.2007.06.002

[13] Hage, C., Rausch, V., Giese, N., et al. (2013) The Novel C-Met Inhibitor Cabozantinib Overcomes Gemcitabine Resistance and Stem Cell Signaling in Pancreatic Cancer. Cell Death \& Disease, 4, e627. http://dx.doi.org/10.1038/cddis.2013.158

[14] Vaz, A.P., Ponnusamy, M.P., Rachagani, S., Dey, P., Ganti, A.K. and Batra, S.K. (2014) Novel Role of Pancreatic Differentiation 2 in Facilitating Self-Renewal and Drug Resistance of Pancreatic Cancer Stem Cells. British Journal of Cancer, 111, 486-496.

[15] Hong, S.P., Wen, J., Bang, S., Park, S. and Song, S.Y. (2009) CD44-Positive Cells Are Responsible for Gemcitabine Resistance in Pancreatic Cancer Cells. International Journal of Cancer, 125, 2323-2331.

[16] Ischenko, I., Seeliger, H., Jauch, K.W. and Bruns, C.J. (2009) Metastatic Activity and Chemotherapy Resistance in Human Pancreatic Cancer-Influence of Cancer Stem Cells. Surgery, 146, 430-434. http://dx.doi.org/10.1016/j.surg.2009.05.003

[17] Li, C., Lee, C.J. and Simeone, D.M. (2009) Identification of Human Pancreatic Cancer Stem Cells. Methods in Molecular Biology, 568, 161-173. http://dx.doi.org/10.1007/978-1-59745-280-9_10

[18] Niess, H., Camaj, P., Renner, A., et al. (2014) Side Population Cells of Pancreatic Cancer Show Characteristics of Cancer Stem Cells Responsible for Resistance and Metastasis. Targeted Oncology, 10, 215-227.

[19] Hung, S.W., Mody, H.R. and Govindarajan, R. (2012) Overcoming Nucleoside Analog Chemoresistance of Pancreatic Cancer: A Therapeutic Challenge. Cancer Letters, 320, 138-149. http://dx.doi.org/10.1016/j.canlet.2012.03.007

[20] Shah, A.N., Summy, J.M., Zhang, J., Park, S.I., Parikh, N.U. and Gallick, G.E. (2007) Development and Characterization of Gemcitabine-Resistant Pancreatic Tumor Cells. Annals of Surgical Oncology, 14, 3629-3637. http://dx.doi.org/10.1245/s10434-007-9583-5

[21] Yan, X.D., Li, M., Yuan, Y., Mao, N. and Pan, L.Y. (2007) Biological Comparison of Ovarian Cancer Resistant Cell Lines to Cisplatin and Taxol by Two Different Administrations. Oncology Reports, 17, 1163-1169. http://dx.doi.org/10.3892/or.17.5.1163

[22] Castellanos, J.A., Merchant, N.B. and Nagathihalli, N.S. (2013) Emerging Targets in Pancreatic Cancer: EpithelialMesenchymal Transition and Cancer Stem Cells. OncoTargets and Therapy, 6, 1261-1267. 